\title{
DEEP DEFECTS IN LOW-TEMPERATURE GaAs
}

\author{
K.P. Korona, J. Muszalski, M. KamińsKa \\ Institute of Experimental Physics, Warsaw University \\ Hoża 69, 00-681 Warszawa, Poland \\ AND E.R. WEBER \\ University of California, Berkeley, CA 94720, USA
}

\begin{abstract}
Conductivity of $\mathrm{GaAs}$ layers grown by molecular beam epitaxy at low substrate temperature $\left(190-200^{\circ} \mathrm{C}\right)$ and then annealed at few different temperatures (between 300 and $600^{\circ} \mathrm{C}$ ) were studied. It was confirmed that electron transport is due to hopping between arsenic antisite defects. Parameters describing hopping conductivity and their dependence on temperature of annealing are discussed. Other deep defects with activation energies of 0.105 , $0.30,0.31,0.47,0.55 \mathrm{eV}$ were found using photoinduced current transient spectroscopy measurements.
\end{abstract}

PACS numbers: 71.55.-i, 72.80.Ey

Molecular beam epitaxy (MBE) GaAs layers grown at low substrate temperatures (about $200^{\circ} \mathrm{C}$, so-called low-temperature (LT) GaAs) demonstrate very interesting properties from both application and scientific points of view. They were successfully used for improvement of electric insulation in GaAs integrated circuits. In science, they attract much attention because of their off-stoichiometry, significant concentration of arsenic antisite defect and hopping conductivity between deep defect centers [1].

In the present paper systematic transport studies of LT GaAs were performed. Layers numbered 3-1871, 3-1872, 3-2088 were grown on a semi-insulating substrate under As rich condition at $190^{\circ} \mathrm{C}$ at MIT Lincoln Laboratory. LT GaAs layers of the 3-1871 and 3-1872 samples were $2.5 \mu \mathrm{m}$ thick. The 3-2088 sample had a $2.0 \mu \mathrm{m}$ of LT GaAs layer insulated from the substrate with a thin $0.5 \mu \mathrm{m}$ layer of AlAs. All samples were divided into parts which were annealed in MBE system at different temperatures.

Optical absorption measurements were done on 3-1872 samples. Concentrations of EL2-like defects were obtained using Martins calibration curve [2].

Temperature-depending conductivity measurements were carried out in the range $12-300 \mathrm{~K}$. It was found that in temperature below $300 \mathrm{~K}$ the conductivity had a hopping character. For each sample an equilibrium ("dark") current was registered. Then, the sample was illuminated with the light $\lambda=0.95 \mu \mathrm{m}$ for ten minutes at low temperature (about $12 \mathrm{~K}$ ) and then during heating up to room 
temperature the current was measured again. Illumination was done in order to quench EL2 to the metastable state but it should also cause a nonequilibrium occupancy of the other centers.

Typical results of the measurements are shown in Fig. 1. For the as grown samples the conductivity after illumination increased, for the samples annealed at high (higher than $300^{\circ} \mathrm{C}$ ) temperature a reverse relation was observed. All illumination induced changes disappeared when the sample was heated up to 120 K. For few samples a small peak at low temperature of thermally stimulated current (TSC) was observed. Absence of other TSC peaks is astonishing because high concentrations of defects were expected. It could be explained by a high carrier recombination rate in LT GaAs.

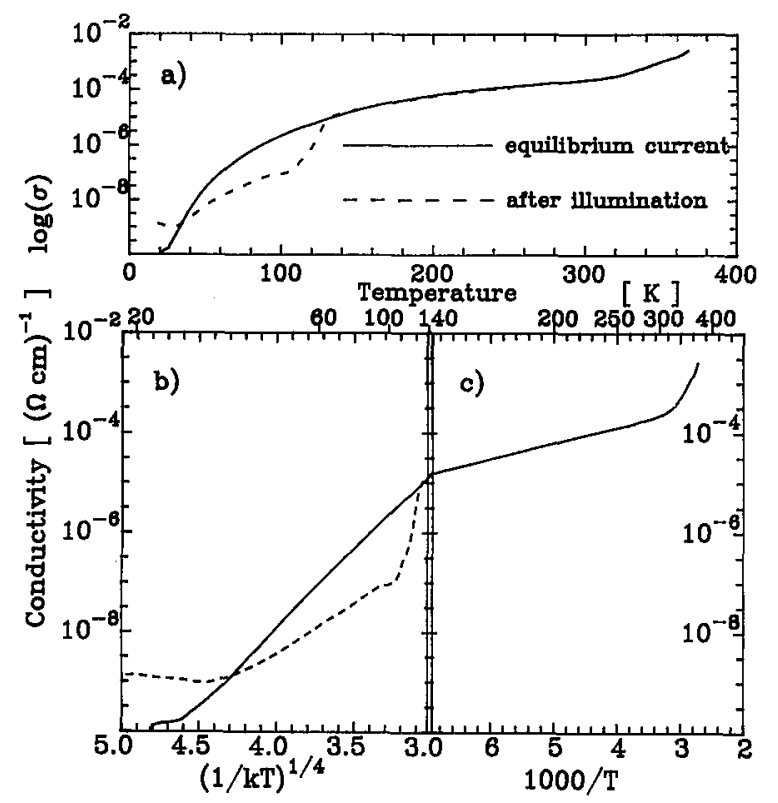

Fig. 1. Typical conductivity versus temperature dependence for LT GaAs layer. The same curve was shown in three different temperatures scales to visualize different characters of the $\sigma(T)$ dependence in low and high temperature ranges.

The hopping conductivity was analyzed basing on percolation theory, taking into account two ranges of temperature. For higher temperature the conductivity $\sigma$ should be

where

$$
\sigma=\sigma_{03} \exp \left(\frac{\varepsilon_{3}}{k T}\right),
$$

$$
\sigma_{03}=\sigma \exp \left(1.73 \frac{2}{a N^{1 / 3}}\right),
$$

$N$ is a concentration of hopping centers, $\boldsymbol{a}$ is a localization parameter of the defect wave function and $\varepsilon_{3}$ is an average energy of Coulomb interaction between two 
impurities. In Fig. 1c logarithm of conductivity versus $1000 / T$ was shown. Fitting the relation (1) to the experimental data, it was possible to determine $\varepsilon_{3}$ for all samples. For annealed samples $\varepsilon_{3}$ decreased with annealing temperature. According to Shklovskii's theory developed for shallow impurities $\varepsilon_{3}$ should increase with $N^{-1 / 3}$ [3]. Basing on EL2 concentration known for some samples from optical absorption measurements, concentrations in the rest of samples were calculated. It was found that concentration of EL2 centers was still high (about $10^{18} \mathrm{~cm}^{-3}$ ) even in annealed samples (up to $600^{\circ} \mathrm{C}$ ). Following Eq. (2), the conductivity $\sigma_{03}$ was examined. From the slope it was found that the wave function localization parameter $a$ was equal to $5.2 \AA$. It is important to notice that such small value of this parameter is characteristic of deep centers.

For lower temperature hopping is characterized by Mott's law

$$
\sigma=\sigma_{0} \exp \left(-\left(T / T_{0}\right)^{1 / 4}\right)
$$

$\left(T_{0}=21.2 /(g(\mu) a), g(\mu)\right.$ is density of states at the Fermi level), which was confirmed by experiment (see Fig. 1b). Fitting the above relation to the experimental data, parameter $T_{0}$ could be determined for all samples. Using the previously found parameter $a$, it was possible to estimate $g(\mu)$ and determine an experimental dependence between the concentration of hopping centers $N$ and the density of states $g(\mu)$. This dependence was used to calculate the concentration of defects after illumination. As a result, it was obtained that in all the samples the concentration of hopping centers decreased after illumination.

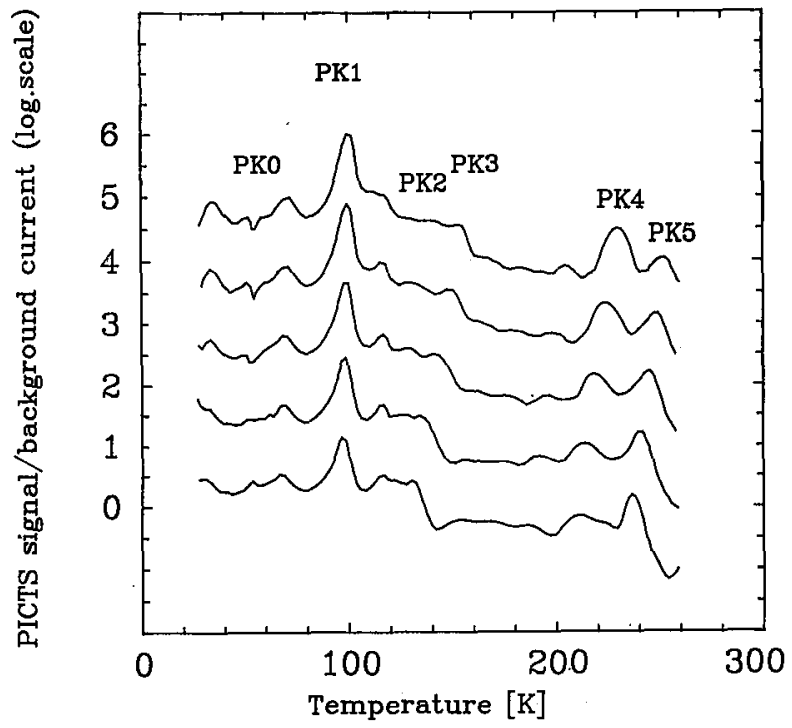

Fig. 2. PICTS spectra of 3-2088 LT GaAs layer annealed at $600^{\circ} \mathrm{C}$. Spectra were measured with different rate windows, every next spectrum was shifted by factor 1 . Each spectrum was divided by the background current. 
In order to find deep defects other than EL2, photoinduced current transient spectroscopy (PICTS) was used. The example of PICTS spectrum is shown in Fig. 2. Both as grown and annealed samples were measured in the temperature range $20-270 \mathrm{~K}$. Current transients observed in as grown samples were strongly nonexponential and thus worthless for further analysis. Nevertheless, the presence of broad peak at about $60 \mathrm{~K}$ was obvious. In annealed samples six PICTS peaks (named PK0...PK5) were registered. First, PK0 peak with activation energy $E_{\mathrm{a}}=$ $0.105 \pm 0.02 \mathrm{eV}$ at temperature about $60 \mathrm{~K}$ could be observed only in samples annealed at temperatures lower than $450^{\circ} \mathrm{C}$. For the rest of samples persistent photocurrent existing in the range $40-100 \mathrm{~K}$ disabled us from observing this peak. The PK1 peak, $E_{\mathrm{a}}=0.47 \pm 0.07 \mathrm{eV}$, at $T=90-100 \mathrm{~K}$ was exactly at the point of disappearance of the persistent photocurrent. Because of its abnormally high activation energy PK1 was not probably connected with electron emission from any point defect. The PK2 and PK3 peaks with $E_{\mathrm{a}}$ of about 0.30 and $0.31 \mathrm{eV}$ observed at $130-150 \mathrm{~K}$ most probably originated from EL6 and EL7 centers known from standard GaAs [4]. The peaks PK4 and PK5 $\left(E_{\mathrm{a}}=0.55 \pm 0.05 \mathrm{eV}\right)$ observed at temperatures $220-260 \mathrm{~K}$ were probably due to emission from deep acceptors. Activation energy of these defects could not be calculated accurately because of quick increase in background current at that temperature.

In summary, hopping conductivity in LT GaAs was examined. Small value of localization parameter $a$ indicated that hopping was via some deep centers. Taking into account that concentration of this center decreased after illumination at $T=12 \mathrm{~K}$ and increased back after annealing at $120 \mathrm{~K}$, the statement that hopping conductivity was due to EL2 centers was confirmed. Other than EL2 defects were found by photoinduced current transient spectroscopy.

\section{- Acknowledgments}

Authors are deeply indebted to F.W. Smith and R. Calawa, MIT Lincoln Laboratory, for supplying us with the LT GaAs layers.

Numerical coefficients in Eqs. (2) and (3) were found by Shklovskii [3].

The work was supported by the Committee for Scientific Research grant number 201799101.

\section{References}

[1] M. Kaminiska, E.R. Weber, in: Proc. 16th Int. Conf. on Defects in Semiconductors, Trans. Tech. Publ., Switzerland 1992, p. 1033.

[2] G. Martin, Appl. Phys. Lett. 39, 747 (1981).

[3] B.I. Shklovskii, A.L. Efros, Properties of Doped Semiconductors, Springer-Verlag, Berlin 1984.

[4] G. Martin, A. Mitonneau, A. Mircea, Electron. Lett. 13, 191 (1977). 